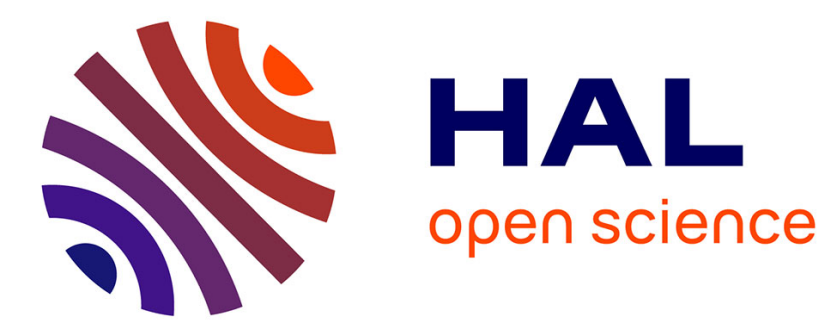

\title{
Pour une étude géographique de la végétation
}

Georges Bertrand

\section{To cite this version:}

Georges Bertrand. Pour une étude géographique de la végétation. Revue Géographique des Pyrénées et du Sud-Ouest, 1966, 37 (2), pp.129-144. 10.3406/rgpso.1966.4544 . hal-02611634

\section{HAL Id: hal-02611634 https://hal.science/hal-02611634}

Submitted on 18 May 2020

HAL is a multi-disciplinary open access archive for the deposit and dissemination of scientific research documents, whether they are published or not. The documents may come from teaching and research institutions in France or abroad, or from public or private research centers.
L'archive ouverte pluridisciplinaire HAL, est destinée au dépôt et à la diffusion de documents scientifiques de niveau recherche, publiés ou non, émanant des établissements d'enseignement et de recherche français ou étrangers, des laboratoires publics ou privés. 


\section{Pour une étude géographique de la végétation}

\section{Georges Bertrand}

\section{Résumé}

Les géographes, quelle que soit leur spécialité, ne peuvent se désintéresser de la végétation. On propose une méthode simplifiée pour l'étude et la représentation graphique des «formations végétales ». En partant des relevés floristiques (ahondance-dominance, sociabilité,) à la fois allégés et aménagés, on construit des « pyramides de végétation ». Du point de vue biogéographique, ces «pyramides » donnent une idée assez précise de la physionomie (stratification, conditions écologiques : pente, sol, etc.) et de la dynamique végétales (climats et sens de l'évolution, concurrence entre les espèces ou entre les strates). Du point de vue géomorphologique, elles mettent en évidence les rapports, en général négligés par les géographes, entre la végétation et la morphogénèse, C'est, avant tout, une méthode de terrain, « approchée » mais rapide, qui permet d'étudier le complexe d'évolution du versant dans toute son intégrité et qui précède normalement des analyses plus fines.

\section{Citer ce document / Cite this document :}

Bertrand Georges. Pour une étude géographique de la végétation. In: Revue géographique des Pyrénées et du Sud-Ouest, tome 37, fascicule 2, 1966. pp. 129-144;

doi : https://doi.org/10.3406/rgpso.1966.4544

https://www.persee.fr/doc/rgpso_0035-3221_1966_num_37_2_4544

Fichier pdf généré le 06/04/2018 


\title{
POUR UNE ÉTUDE GÉOGRAPHIQUE DE I.A VÉGÉTATION
}

\author{
par Georges BERTRAND
}

\author{
"Il serait bon que les géographes \\ se tournent davantage vers les ques- \\ tions biologiques. 》 \\ H. Gaussen, \\ Annales de Géographie, 1957. \\ I, p. 18.
}

L'époque n'est pas tellement éloignée où l'on envisageait l'évolution d'un versant en roche nue, ou recouvert seulement par ses propres débris, sans se préoccuper de l'existence et du rôle possibles de la végétation et $d u$ sol. Les phénomènes biologiques ont pourtant acquis droit de cité grâce aux progrès de la géomorphologie climatique qu'il serait de ce fait, et comme le souhaite .J. Tricart, plus juste d'appeler « bioclimatique » (1). L'apport de la pédologie est particulièrement fécond. On sait, par exemple, ce que la recherche géomorphologique a gagné en sîreté et en finessie grâce à l'interprétation des paléo-sols.

Rien de tel ne s'est passé pour la régétation. Cette situation reflète l'une des plus graves lacunes de la géographie phrsique française : la méconnaissance profonde de tout ce qui touche à la botanique. Certes, les exceptions deviennent nombreuses. Mais ce problème des rapports entre le tapis végétal et la morphogénèse des versants, qui occupe pourtant une place honorable dans l'enscignement. théorique et les grands traités de géographie physique (2), ne se manifeste encore que très timidement au niveau des recherches sur le terrain. Si on laisse de côté la tendance purement biogéographique, qui en est encore à ses premiers débuts, on a l impression que cette

(1) J. Tricakt, Principes et méthodes de la géomorphologie. Paris, Masson, 1965, np. $31-34$.

(2) J. TRICARt et A. Callecy, Introduction d la gemorphologie climatique. Paris. Cours C. D. C. - P. Brot. Precis de géographie physique generale. Paris. Colin, 1959, pp. 123-138 et surtout Formations végetales du globe. Paris, S.E.D.E.S., 1965 
question n'a jamais été réellement posée et n'a suscité aucune réflexion méthodologique.

Les causes de cette désafiection sont multiples ot il $n$ ' $y$ a pas lieu de les analyser ici. On peut cepondant faire remarquer que le rapport régétation-érosion a été trop sourent réduit à un manichéisme des plus frustes où la Tégétation, considérée comme une simple entité, joue l'unique rôle d'ceran protecteur vis-à-v's de l'érosion. Cette conception primitive appelle deux critiques. I) abord, la régétation n'est pas une simple « couverture » protectrice. ("'est un milieu vivant dont las rapports arec lérosion se placent au niveau d'interactions infiniment complexes. Hnsuite, la régétation revêt des aspects multiples et plus ou moins stables, qu'on ne peut éroquer qu'après une enquête botanique préalable, aussi bien floristique qu'écologique. Or, hoaucoup de géographes sont, non sans raison, rebutés par le long apprentissage taxonomique indispensable pour analyser, même sommairement, le moindre tapis végétal. A ce propos, il faut regretter que la formation du géographe so:t si éloignée de colle du naturaliste (3).

Il paraît dence intéressant de présenter ici une méthode d analyse sommaire de la végétation. Elle emprunte heaucoup aux méthodes classigues des relevés de régétation des phytogéographes; mais elle a été adaptée et complétée pour mieux répondre aux préoccupations des biogéographes et des géomorphologues. C'est, arant tout, une méthode de terrain. Eille a été utilisée, non sans résultats, par des équipes d'étudiants de Diplômes d'Etudes Supérieures de l'Institut de Géographie de Toulouse qui n'avaient, au départ, aucune formation botanique spéciale (4).

\section{1. - IAS 《YRAMHDE DE VÉGÉTATION 》.}

Il faut d'abord choisir l'unité régétale qui servira de base à l'étude du tapis végétal. $I^{\prime}$ ' « association régétale » définie par Braun-Blanquet et les phytosociologues est trop complexe du point de vue floristique et trop peu significative du point de rue écologique. Le choix s'est done porté sur la «formation végétale » des phytogéographes (5). Elle se définit comme un groupement de

(3) Cette orientation de recherche aurait éte impensable sans lappui constant du Service de la Carte de la Vététation de la France (C. N. R. S.) et du Centre de troisième cycle de Biogéographie de la faculté des sciences de Toulouse. Nous remercions plus spécialenent le Professeur P. Rey, le I'rofesseur ( $x$. Dupias, inlassable dans ses conseils, et notre ami M. Delpoux, maitre-assistant agrégé.

(4) Nous les remercions très amicalement de leurs suggestions et de leurs critiques.

(5) H. Gausser, Geographie des plantes. Paris, Colin, 1954, pp. 105-127. ... P. OzendA, Biogeograpte végetale. Paris, Doin, 1964, pp. 247-250. 
végétaux qui présente, malgré des différences entre less espècess, des caractères biologiques et un faciès analogues (exemple : la torêt, la lande). Malgré son imprécision floristique, elle peut être retenue comme point de départ de lanalyse de la végétation car elle correspond à un aspect du paysage directement sensible au géographe. Isa solution adoptée eonsiste à parrenir, par l intermédiaire de la classique méthole des relevés floristiques, a une représentation glarphique qui soit à la fois structurale (aspect phrsionomique : hauteux, densité, stratification; composition floristique), dronamique (état actuel d'équilibre et sens de l'évolution) et. enfin. qui soit comparable d'une tormation régétale à l'autre.

1. Les relevés floristiques et physionomiques. - i. Abomelance dominance ot sociabiliti des plantes. - les botanistes recoment it la méthole dite des "relevés floristiques » (6). On choisit sur le trovain un secteur représontant l'état moxen de la formation vécétalo à étudier et on délimite lan cercle denviron $10 \mathrm{~m}$ de rayon afin que les relevés soient comparables entre eux. On peut alors établir. Ia liste des plantes qui se treuvent à lintérieur du périmètre considéré, en notant pour chacume dentre elless, dime part, lat surtace couverte, d'autre part, le mole de groupement. Le travail de elassement est simplifié si on adopte une échelle de 0 à 5 :

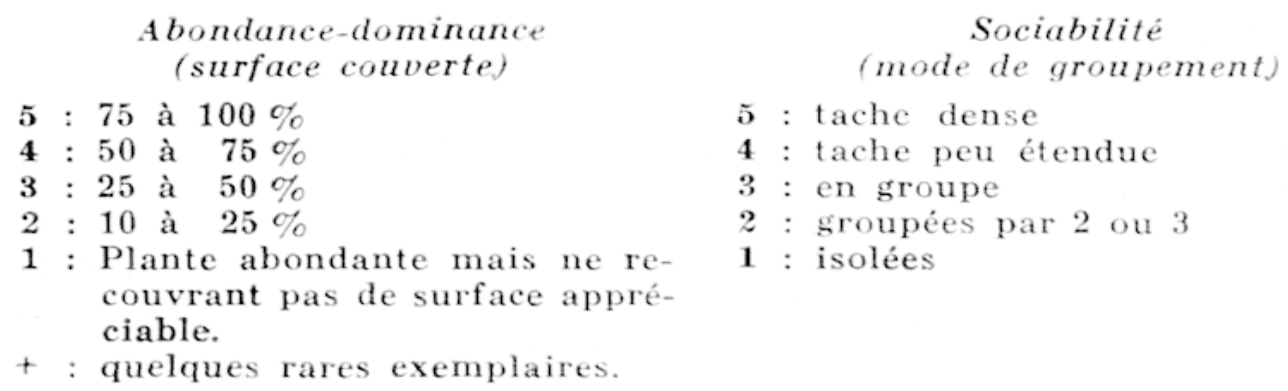

Malgré son apparente imprécision, ente méthode dome d'execllents résultats. surtout quand elle est maniéc aree un peu d habitude.

Ce premier travail est de loin le plus délicat, ear il suppose do bennes connaissances taxonomicues. S'il n'est pas possible de faire appel à un botaniste, il faut essayer de trancher la difficulté dès le départ en distinguant trois prandes catégories de plantes :

- les espèces phrsionomiques qui participent à la compasition dn paysage et que tout géographe se doit de comnaitre. Pour la garrigue méditerranéenne citons par exemple : le Chêne Vert, le Chêno kermès, le Romarin, le Brachypode ramoux, ete.

(6) Exposé conmode des méthodes classiques dans P. Camiss, Giographie botanique, Paris, 1'. L. F., 1948. Coll. "Que sais-je?". 
- les plantes dites « indicatrices », en général peu nombreuses et peu risibles, qui renseignent sur l'état d'équilibre de la formation et sur sa dynamique interne. La présence de quelques pieds de Blechum spicant ou de I)aphné des bois (Daphne laureola L.) dans une lande de l'étage montagnard prouve que cette lande est proche do l'état forestier ot peut facilement être reboisée. On peut négliger ces détails si on ne s’intéresse qu’à la physsionomie actuclle de la végétation, mais on passe ainsi à côté de précieuses indications dynamiques.

- il reste enfin toutes les autres plantes, c'est-à-dire les ubiquistes (par exemple la Bourdaine (Rhamnus frangula L.) dans le Midi Aquitain), et les espèces très spécialisées qui colonisent des biotopes limités, bien distincts de la formation proprement dite - plantes des rochers et des murs comme le Polypode commun ou la Rue des murailles (Asplenium ruta muraria L.). On peut, sans trop d'inconrénients, les ignorer.

B. La stratification du tapis ıégétal. - Il faut aussi classer les plantes d'après leur taille, c'est-à-dire les répartir par strates :

\begin{tabular}{|c|c|}
\hline 5. strate arborée & $\begin{array}{l}\text { Manteur moyenne } \\
\text { au-dessus de } 7 \mathrm{~m} \text {. }\end{array}$ \\
\hline 4. strate arborescente & 3 à $7 \mathrm{~m}$. \\
\hline 3. strate arbustive & à 3 \\
\hline 2. strate sous-arbustive & 0,50 à 1 \\
\hline $\begin{array}{l}\text { 1. strate herbacée } \\
\text { et strate muscinale confondues }\end{array}$ & à $0,50 \mathrm{~m}$. \\
\hline
\end{tabular}

Nous arous ajouté, enfin, une dernière opération simple mais capitale. Elle consiste à appliquer l'échelle de l'abondance-dominance non plus aux différentes espèces mais à chacune des strates. Cela permet de définir commodément la stratification du tapis végétal (nombre de strates, rapport de recourrement des différentes strates). C'est l'origine des « prramides » de végétation.

Dans la pratique, tous ces relevés seffectuent en même temps et ne demandent que quelques minutes (ef. la fiche-type et la fig. 2). Il vaut mieux effectuer plusieurs relevés à l'intérieur d'une même formation pour déterminer sa dỵamique et définir les modes de contact arec les formations roisines.

2. La construction des " pyramides" de végétation. - Commenter une liste floristique et la comparer à une autre est une opération délicate surtout pour un non-botaniste. Mais la plupart des difficultés tombent d'elles-mêmes si on porte les résultats de l'analyse phissionomique et floristique sur un graphique de type 
pyramide (fig. 1). Sur un papier millimétré, on prend un segment de droite horizontal de $10 \mathrm{~cm}$ de long. Sur cette base et en son milieu, on dresse perpendiculairement laxe de la prramide. On dispose les strates de régétation symétriquement par rapport à l'axe en tenant compte de leur ordre normal de superposition, de leur ind ce de recourrement (abondance-dominance $1=1 \mathrm{~cm}, 2=2 \mathrm{~cm}$, $5=\overline{\mathrm{cm}}$ ) et de leur épaisseur. On peut indiquer l'épaisseur réelle de chaque strate, mais cela introduit de sérieuses eomplications sans apporter des précisions intéressantes. On a choisi les intervalles suivants, arbitraires mais commodes : strate $1=0,5 \mathrm{~cm}$, strate 2 et $3=1 \mathrm{~cm}$, strate $4=1,5 \mathrm{~cm}$, strate $5=\underline{\mathrm{cm}}$ ot plus, suirant les cas.

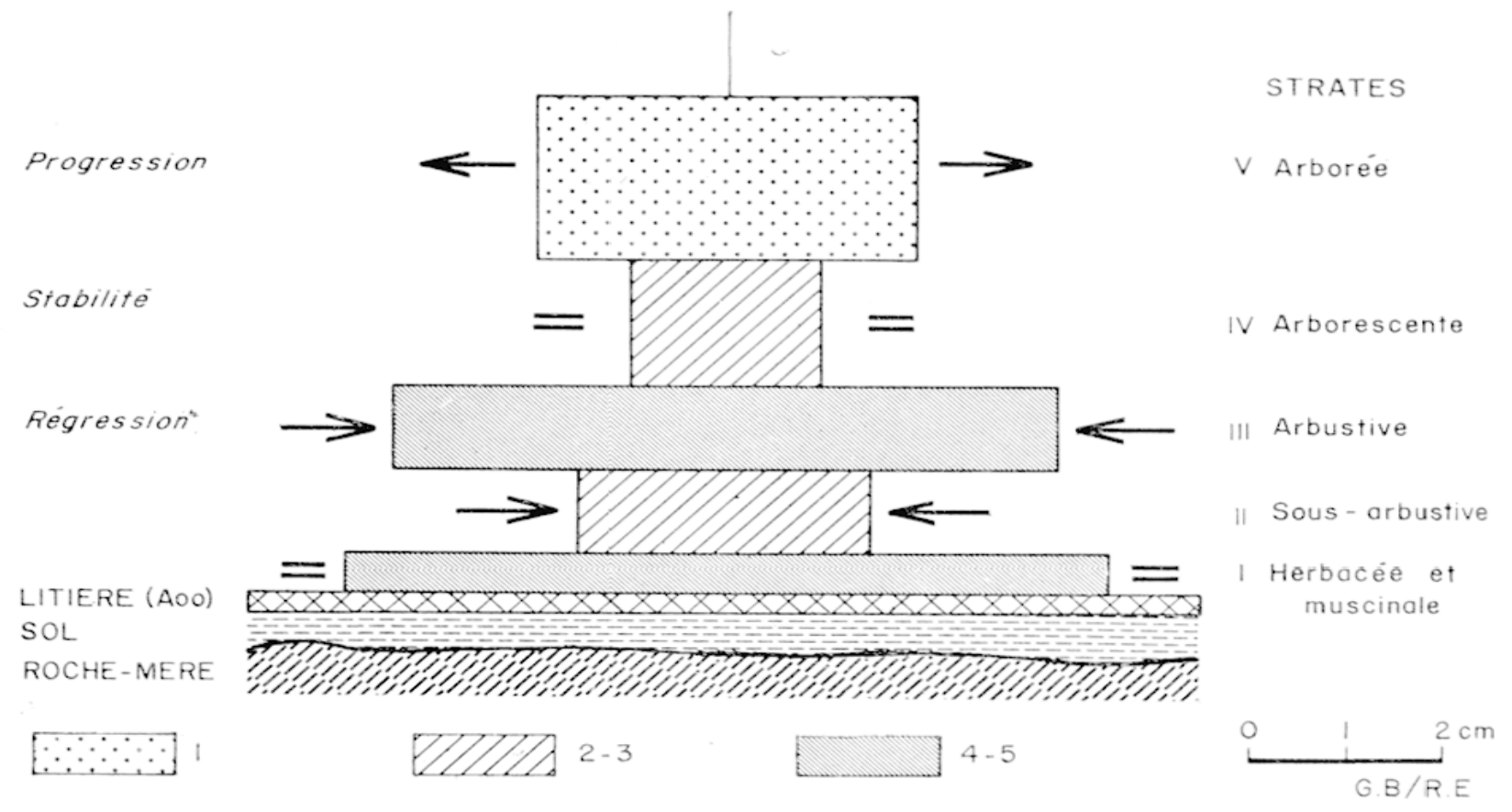

FIG. 1

Pyramide de végétation-type.

Mode de groupement des plantes à lintérieur dine môme strate : 1. pieds isolés. - 2-3. par touffes. - 4-5. par masses compactes.

On complète cette pyamide en $y$ reportant des indications qui peuvent varier suivant lobjet de l'étude :

- à la base, il faut placer la litière (épaisseur, surface couverte), car cette dernière joue un rôle essentiel dans léquilibre de la formation. Au-dessous, on indique le trype de sol (épaisseur, profil simplifié) et la roche-mère. Il suffit de faire basculer la proamicle d un angle égal à la valeur de la pente du versant sur lequel se trouve la formation pour donner une image assez précise des conditions stationnelles (pente, insolation, ruissellement. et ressuyage) (fig. 2.). 
- les strates peurent porter les indications suivantes sous forme de « plages » : ou le mode de groupement des plantes de la strate sans faire de distinction entre les plantes (échelle de 0 à 5 comme pour la sociabilité des espèces) (fig. 1) : ou les variations sa isomnières de la densité des différentes strates, ce qui est important pour distinguer la végétation sempervirante de la caducifolice ou pour montrer le décalage dans le crole végétatif entre les différentes strates d'une forêt (régétation prévernale des sous-bois).

- enfin, on peut décaler les strates par rapport à l'axe de la pyramide pour opposer des eatégories différentes de plantes (fig. 7 et 8). Des flèches indiquent lo sons ot la rapidité de l'érolution des différentes strates.

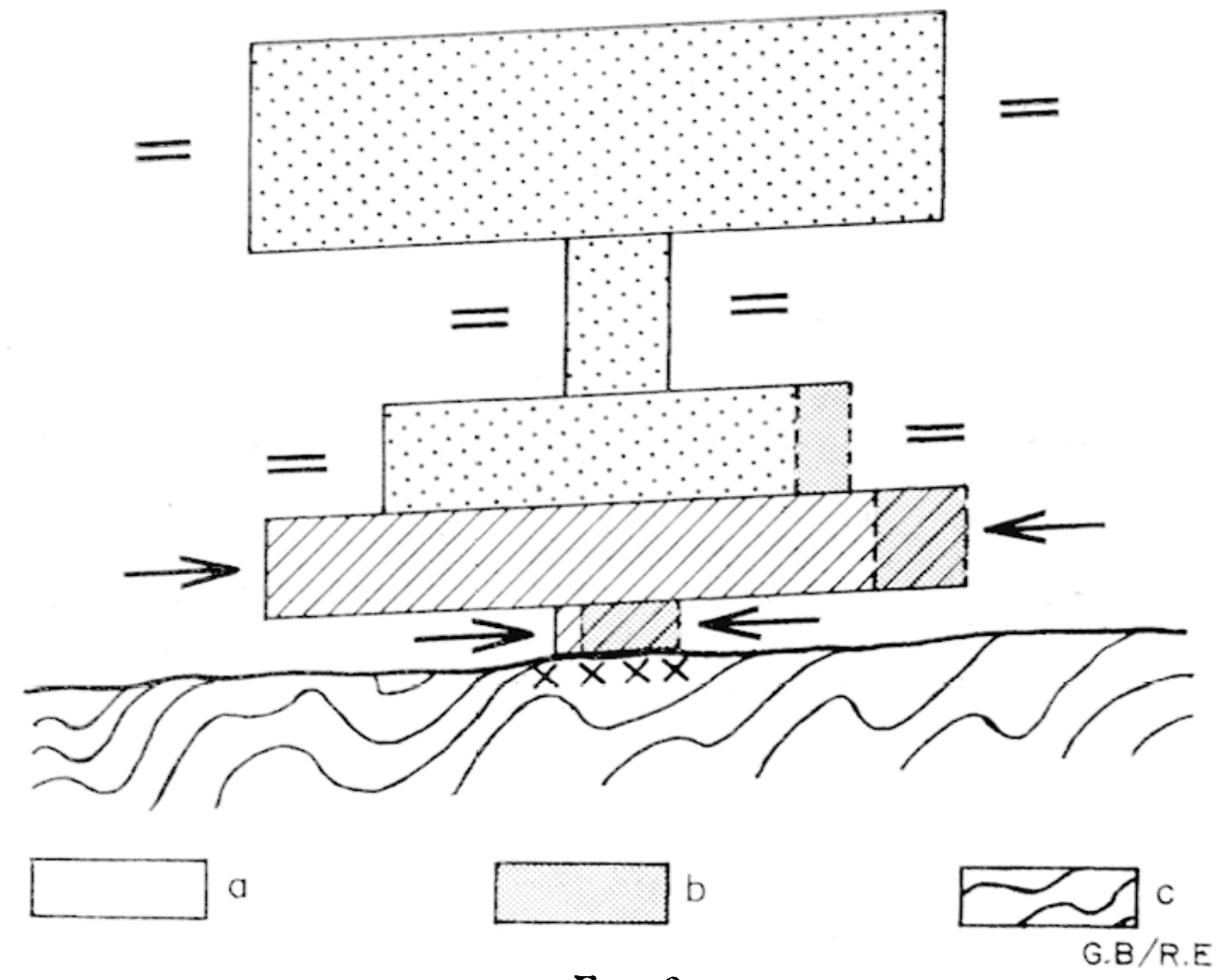

FiG. 2

Forêt claire à Chêne Vert d'Allende (Cf. fiche biogéographique type) a. plantes méditerranéennes. - b. plantes atlantiques. - c. schistes.

Il y a bien dautress possibilités. mais il vaut mieux que le schéma comserre sa simplicité première afin que l interprétation soit immécliate. Il existe d'autres méthexles plus complexes et plus précises. surtout du point de vue érologique et floristique : nous pensons mar exemple aux tentatives de $P^{2}$. I)ansereau pour représenter la structure de la récétation a partir des types biologiques de Raunkiarer $(T)$.

(7) P' Daxisereal. Bingeography, an emlogical perspectine. New-York, 1957,
of. $117-153$. 


\title{
FORET CLAIRE A CHENE VERT D'ALLENDE
}

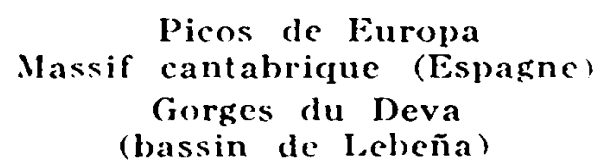

\author{
Domaine mediterranén \\ intramontagnard \\ Séric du Chêne Vert \\ arec relictes de Chêne-liège
}

\begin{tabular}{|c|c|c|c|}
\hline \multirow{2}{*}{ STRATE } & \multicolumn{2}{|c|}{ PAR ESPÈCE VÉGÉTALE : } & \multirow{2}{*}{$\begin{array}{l}\text { PAR STRATE : } \\
\text { Abondance- } \\
\text { dominance : }\end{array}$} \\
\hline & $\begin{array}{l}\text { Abondance- } \\
\text { dominance : }\end{array}$ & Sociabilité : & \\
\hline $\begin{array}{l}\text { 5. Chêne Vert. . . . . . . . . . . . . . . . } \\
\text { Chêne-Liège. } \\
\text { Arbousier (A. unedo) . . }\end{array}$ & $\begin{array}{l}3 \\
+ \\
+\end{array}$ & $\begin{array}{l}1 \\
1 \\
1\end{array}$ & 3 \\
\hline 4. Filaire (Ph. media)........ & + & 1 & 1 \\
\hline $\begin{array}{l}\text { 3. Térébinthe. } \\
\text { Jasmin arbris. (J. fruticans) } \\
\text { Aubépine (C. monogyna).... }\end{array}$ & $\begin{array}{l}1 \\
1 \\
+\end{array}$ & $\begin{array}{l}1 \\
1 \\
1\end{array}$ & 2 \\
\hline $\begin{array}{l}\text { 2. Ciste à feuilles de sauge.... } \\
\text { Salsepareille (Smilax aspera) } \\
\text { Genêt occidental (Genista } \\
\text { hispanica ssp. occidentalis) } \\
\text { Héllébore fétide............ } \\
\text { Chèvrefeuille d'Etrurie (Loni- } \\
\text { cera etrusca). ........... } \\
\text { Bruyère cendr. (Erica cinerea) }\end{array}$ & $\begin{array}{l}2 \\
1 \\
\mathbf{1} \\
+ \\
+ \\
+ \\
+\end{array}$ & $\begin{array}{l}3 \\
1 \\
1 \\
1 \\
1 \\
1 \\
1\end{array}$ & 3 \\
\hline 1. Graminées diverses. & 1 & 2 & 1 \\
\hline
\end{tabular}

0. Litière discontinue et mal décomposée.

Altitude : $380 \mathrm{~m}$. Pente : $5 \%$ Exposition : Soulane SE

Climat : Méditerranéen d'abri, $\mathrm{P}<2 \mathrm{~T}$ en juillet-août.

Précipit/ann $/ \mathrm{moy}=600 \mathrm{~mm}$ environ.

Microclimat : Très abrité contre paroi calcaire $(1800 \mathrm{~m})$.

Roche-mère: schistes de faciès Culm et gros éboulis de calcaire cristallin.

Sol : Discontinu, sous les racines. Protorendzine.

Action anthropique: Forêt très dégradée, parcours des chèvres et des moutons.

Erosion : Sol décapé, éboulis localement actif, peu de ravinements.

Dynamique d'ensemble: Forêt relicte qui se régénère mal et qui se maintient sur place par sa seule présence.

Type de fiche biogéographique de terrain (cf. fig. 2). 
La pyramide de régétation donne de la formation végétale une image, certes grossière, mais eependant significative. A la fois structurale et dynamique, elle facilite grandement les comparaisons d'une formation à l'autre. En dépit de sa conception rudimentaire, clle permet de micux définir le complexe géographique dans lequel s'insère la formation végétale et de déboucher sur des explications sýnécologiques au sens le plus large de ce terme.

Pour l'instant, il n'est pas possible de tirer toutes les conséquenees de cette méthode. On se contentera de l'appliquer à deux domaines presque confondus dans la réalité mais arbitrairement séparés par le cloisonnement des sciences : la biogéographie et la géomorphologie. Ce ne sont que de simples remarques, repasant. sur un petit nombre d'échantillons limités au secteur atlantico-méditerranéen.

\section{II. - INTERLRÉTATION BIOGÉOGRAPHIQLE :}

RSQTISSE STRICTCRAIF ET DYNAMIQUE ID LA VÉGÉTATION.

La pyramide donne du tapis végétal une image suffisamment expressive pour (qu il vaille la peine de beancoup insister. Il suffit de comparer quelques prramides. Une forêt (fig. 3) se distingue parfaitement d'une lande (fig. 5) ou d'une garrigue (fig. 6) par l'importance relative de ses strates. L'ne futaie « régulière » à sousbois clairsemé comme la Hêtraie de Leyme (fig. 3) présente une structure trop différente de la Subéraic de Llauro à sous-bois envahissant et à strate arborée ouverte (fig. 4) pour que l'on puisse confondre ces deux milieux forestiers. Par contre, il y a une évidente parenté entre cette forêt de Chêne-Liège et la forêt claire de Chêne vert d'Allende (fig. 2). Les formations ouvertes comme la garrigue à Cenêt Scorpion ot Lavande vraie de Cervera-de-Pisuerga (fig. 6) s'opposent aux formations fermées du type de la lande sub-atlantique du littoral asturien où les Ajones, les Ericacées et les Fougères s'entrelacent vigoureusement (fig. 5).

En partant d'une telle analyse de la structure régétale, il est done facile de classer les formations d'après le mode de recouvrement, en faisant intervenir aussi bien le nombre de strates (mono ou pluri-strates), que la strate dominante, ou que le mode de groupement des plantes (tapis continu ou vacuolaire, coussinets). On donne ainsi une meilleure hase structurale aux classifications traditionnelles (Schimper-Faber, Allorge-.Jovet) (8), et on débouche directement sur la cartographie de la végétation.

(8) Présentation succinte dans Ozenda, ouvr. cité, pp. 248-249. 

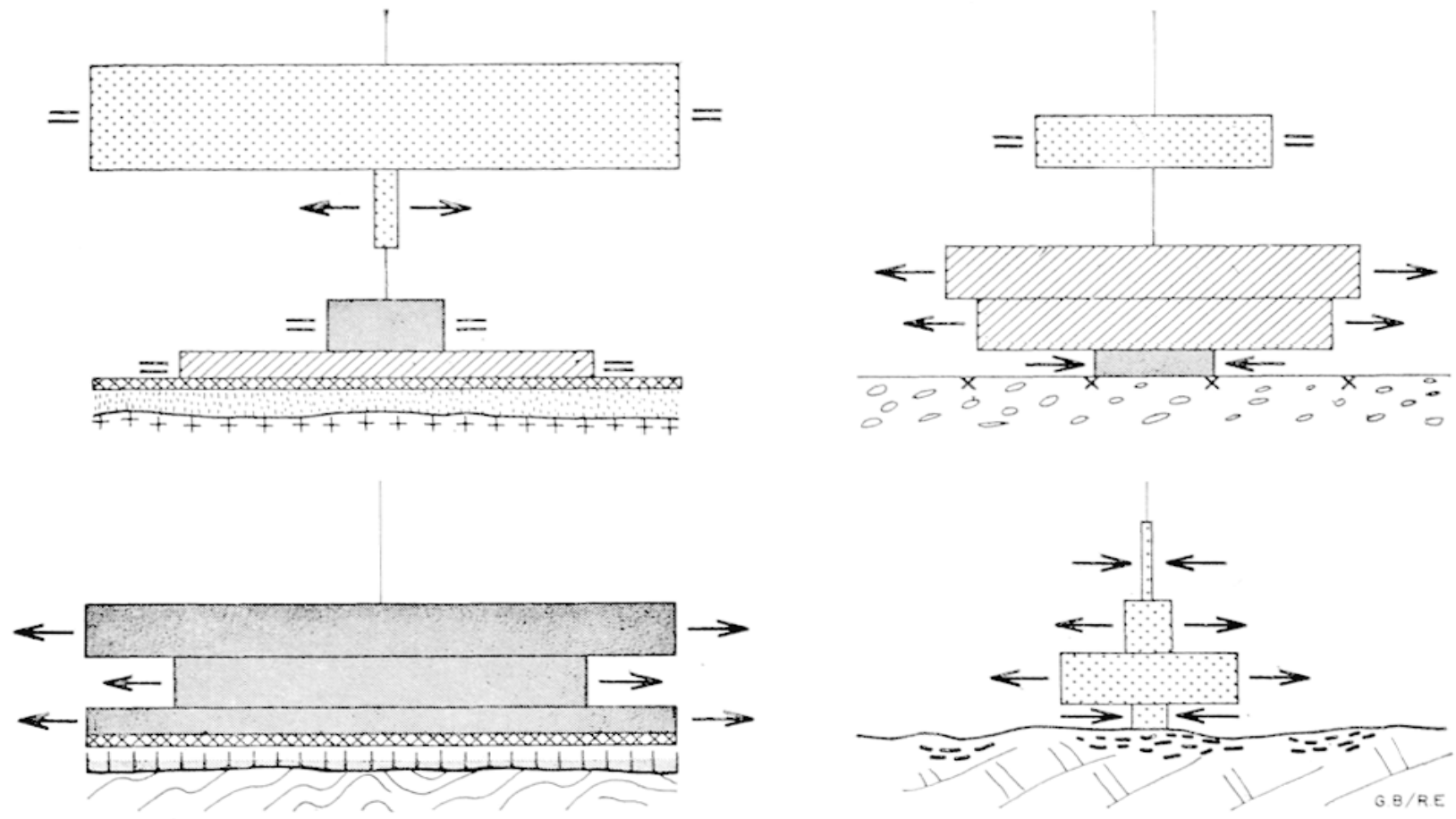

Fig. 3 ien haut, à gauche)

Hêtraie «régulière 》de Leyme (Quercy oriental, France).

1. Blechnum spicant, Aspidium aculeatum. - 2. Myrtille en touffes. 4. Jcunes Hêtres. - j. 100 \% de Hêtres. - Sol brun forestier à Mull sur arène granitique. - litière épaisse.

Fig. 4 (en haut, à droite)

Subéraie claire (incendiée) de Llauro (Aspres du Roussillon, France).

1. Brachypode rameux, Lavande stoechas. - 2. Ciste de Montpellier, Genêt Scorpion. - 3. Bruyère arborescente, Oxycèdré - 5. Chêne-Liègé à $100 \%$.

Fig. 5 (en bas, à gauche)

Lande haute subatlantique Ribadesella (littoral asturien, Espagne).

1. Graminées. Mousses. - 2. Ericacées. - 3. Ajonc d'Europe et Fougère Aigle. - Sol brun hydromorphe sur schistes.

Fig. 6 (en bas, à droite)

\section{Garrigue sur calcaire (Mariserrana - Cervera de Pisuerga, Vieille Castille, Espagne).}

1. Graminées et Arbousier rampant. - 2. Lavande vaice Genêt Scorpion. -3. Oxycèdre. - 4. Chêne Vert. Argile de décalcification en poches.

Les numéros correspondent aux différents strates, cf. fig. 1.

Mais c'est surtout l'interprétation dynamique qui doit retenir l'attention du biogéographe. Les prramides traduisent parfaitement la concurrence entre les espèces. Dans les «landes-maquis » du littoral 
asturo-cantabrique (fig. T), des espèces sub-méditerranéennes sont en concurrence aver des plantes sub- et eu-atlantiques. Les premières. surtout somprerirantes et ligneuses (Chêne Vert, Arbousier, Prunier llahaleb, Filaibe. Térébinthe ete), composent les strates slupéricures. las serondes, des Ajones, des Bruyeres et des Ciaminées, envahissent les niveaux inférieurs et etouffent les plantules des espèces supérieures. La ('hênaic méditerranéenne est lentement colonisée par la lande atlantique. C'est le type même de la formation relicte en roie de réserssion (9).
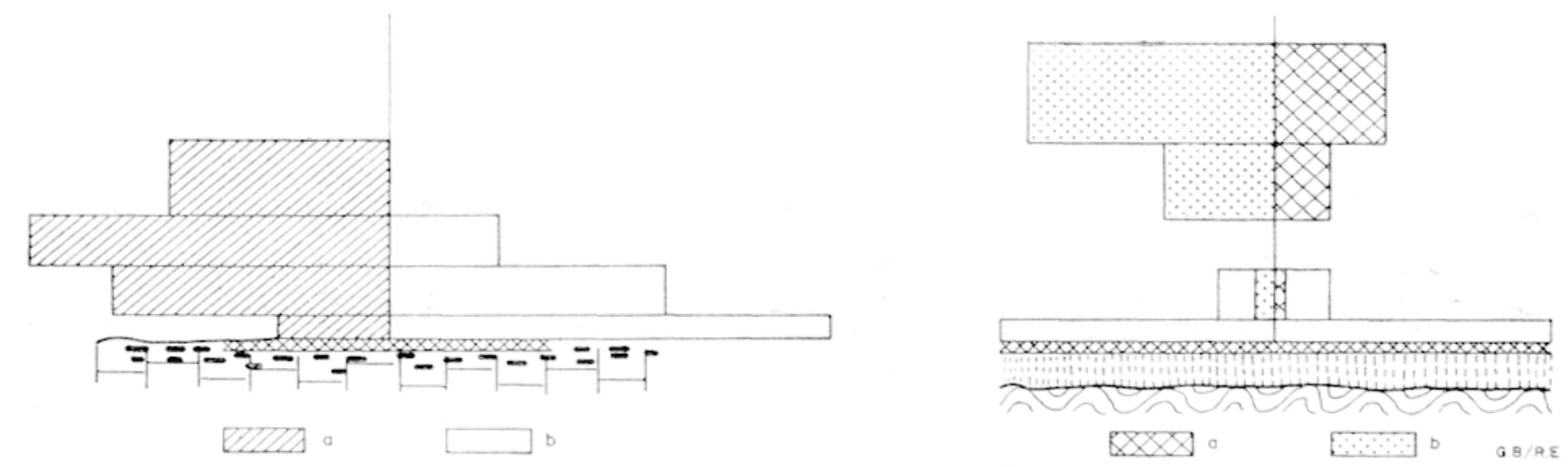

Fig. 7 (à gauche)

«Lande-garrigue» de la « rasa 》de Pechón (littoral cantabrique, Espagne). a. Plantes méditerranéennes ou sub-méditerranéennes : Chêne Vert. Arbousier, Salsepareille. etc. - b. plantes atlantiques: Ajonc d'Europe. Bruyères variées. - Sol : argile de décalcification.

Fig. 8 (à droite)

\section{Châtaigneraie de Zardón (Sierra de Cuera, Asturias, Espagne).}

a. Chêne pédonculé. - b. Châtaignier.

Dans la vallée du Kardón (Sierra de ('uora, Asturias), le Châtaignier, introduit puis abandomné par l homme. envahit. la Chênaie semi-montagnarde humide (Sessile et surtout Pédonculé) (fig. 8). Grâce à un recépage rigomenx, il gagne de vitesse toutes les autrex espèces ligneuses. Ine Châtaigneraie sub-spontanéc se substitue assez rapidement à la Chênaie climacique. Les Chênes ne jouent qu'un rôle subordonne, par contre lo sous-bois (Myrtille, Capillaire noire) et le sol (brun lessivé) n'ont pas évolué. Il y a eu seulement substitution de lespèce dominante.

Les pvramides permettent également de suivere la concurrence entre les différentes strates. Il suffit de reprendre les exemples 3 et 4.

(9) G. Bertrand, Les formations vegḱtales méditerranéennes du versant nordcantabrique (Espagne du Nord-Ouest) : "n probleme erologique. Rull. Assor. Géogr. Francais. 14tit, no $328-329$, pp. 12-iti. 
Dans la Hêtraie de Leyme, cest le triomphe absolu de la strate arborée, continue et dense, qui en filtrant la lumière limite l'extension du sous-bo:s, où l'on ne rencontre que des espèces sciaphiles ou hygrophiles comme les Fougères (Blechum spicant. Aspidium aculeatum) ou bien des plantes prérernales comme l'Anémone Sylvie at l'Aspérule odorante. Par contre, sous le feuillage clair des ChênesLièges des Aspres du Roussillon, se développe une strate arbustive autonome composét de xérophiles et d'héliophiles.
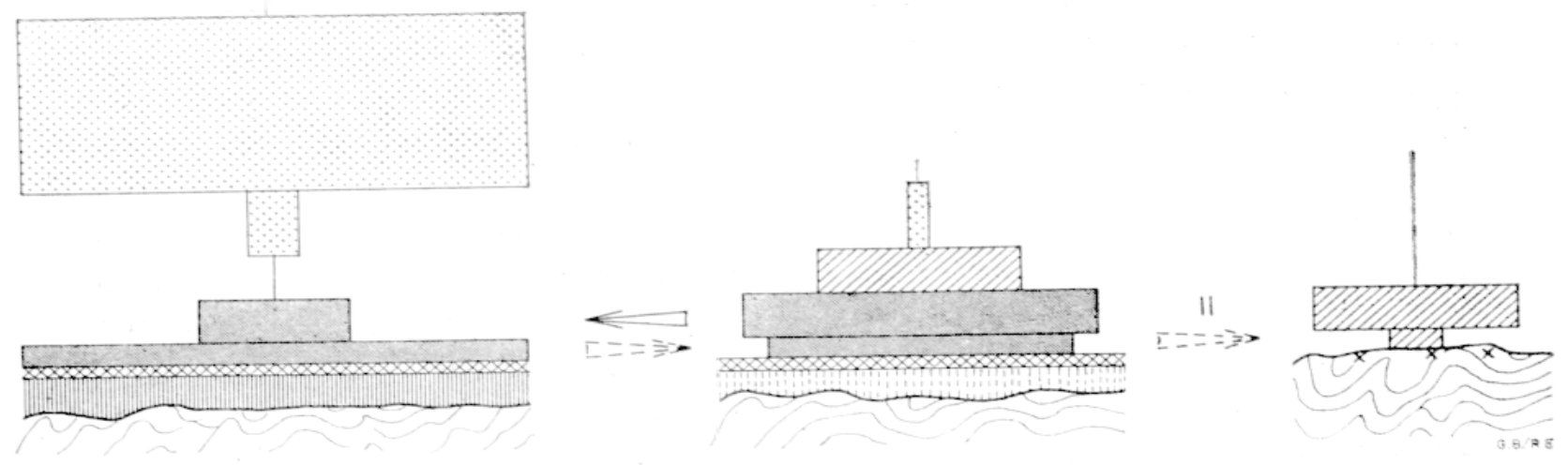

FIG. 9

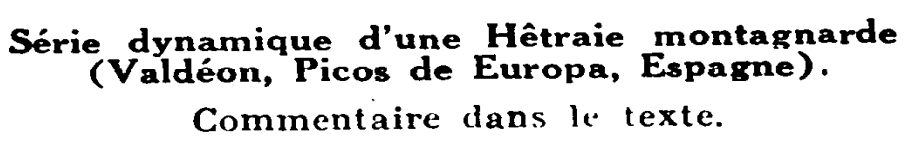

Enfin, les pyramides permettent de mieux suivre l'évolution à l'intérieur d'un même domaine biogéographique. Prenons le cas de la Hêtrạe de Valdeón (Picos de Furopa, Massif cantabrique). On constmit. autant de promides qu'il $r$ a de formations végétales comprises daus ce domaine. Pour simplifier, on $n$ 'en retiendra que trois (fig. 9) : la futaie plésioclimacique sur sol brun forestier à Mull, la lande haute à Genêt condré et Sarothamne cantabrique sur $\therefore$ ol hrun lessivé à Moder, enfin la lande-pelouse ouverte à Bruyère cendrée et à Callune sur des sols bruns dégradés et des Rankers d'érosion. l'évolution de la végétation et celle des sols sont donc. plus ou moins parallèles. Les quelques Allouchiers qui parsèment la lande haute constituent le front pionnier de la forêt. Par contre. lorsque l'évolution régressive atteint le stade de la lande-pelouse xérophile, tout retour au climax parait compromis car les sols sont appauvis et les pentes livrées à des érosions pelliculaires de type décapant. 


\section{III. - INTERPRÉtATION GÉNOMPHOLOGIQLE :}

LES RAPPORTS FNTRE IA VÉGÉTATION ET L'ÉROSION « BIOLOGIQUE 》.

Parmi les « facteurs écologiques », « correctits écologiques » et autres « facteurs limitants ». les botanistes classent la pente, les sols, les microclimats, ete ; c'est-à-dire des phénomènes qui servent à définir la station végétale ou le biotope comme des milieux stables. Jamais, ou pratiquement jamais, il n'est question de l'érosion. Pour éviter les confusions et les interprétations abusives, il est bon de définir exactement ce terme. Sauf eas exceptionnel, il ne s'agit pas ici de l'érosion « géologique » ou, mieux, « géomorphologique » créatrice du relief, mais d'une érosion de type épidermique qui, tout en ayant des prolongements géomorphologiques évidents mais lointains, n intéresse que la partie la plus superficielle du substratum rocheux et, surtout, la « couverture virante des versants » (P. Birot), c'est-à-dire le sol, la litière et la végétation. Pour cette raison, on peut la qualifier de «biologique». Entre l'érosion «biologique» et l'érasion réomorphologique il n'y a qu'une différonce d'intensité, la seconde étant le prolongement naturel de la première. Mais l'échelle des phénomènes n'est pas la même. On se place ici sur le plan biogéographique qui, du point de vue temporel, est plus proche du plan historique que du plan géologique.

Les prramides de régétation permettent de se faire une meilleure idée des rapports entre la régétation et l'érosion «biologique ». Ou point de vue structural, il est classique d'opposer les formations végétales ouvertes aux formations fermées. La garrigue de Mariserrana (fig. 6) s'ouvre à tous les niveaux. Les plantes isolées ou en touffes laissent entre elles de larges plaques sans sol et sans litière. Les ravineaux et les microglacis de quelques décimètres s'y épanouissent librement. Sur les pentes supérieures à $10 \%$ on passe progressivement à des ravinements d'échelle métrique qui sont déjà une manifestation de l'érosion « géomorphologique ». Isa même érolution se retrouve sous le maquiss à Chêne Vert et à Filaire de Liébana (fig. 10), qui serait pourtant considéré par les botanistes comme une formation fermée. Mais si ce maquis est impénétrable à hauteur d'homme, les strates inférieures sont très réduites et protègent mal des ravinements. Plus net encore est le cas d'un rebo:sement de pins dans la vallée supérieure du Júcar (fig. 11) dans la serranía de Cuenca (Espagne). le reboiscment a réussi et les pins sont d'assez belle venue mais le développement du sous-bois n'a pas suivi. I'n ruissellement de type aréolaire s'est emparé de pentes pourtant faibles et empêche visiblement la germination. Il est clair qu'un tel rehoisement ne doit pas apporter beaucoup de 
modifications aux conditions de l'écoulement des eaux dans le bassin si souvent dévasté du Júcar.
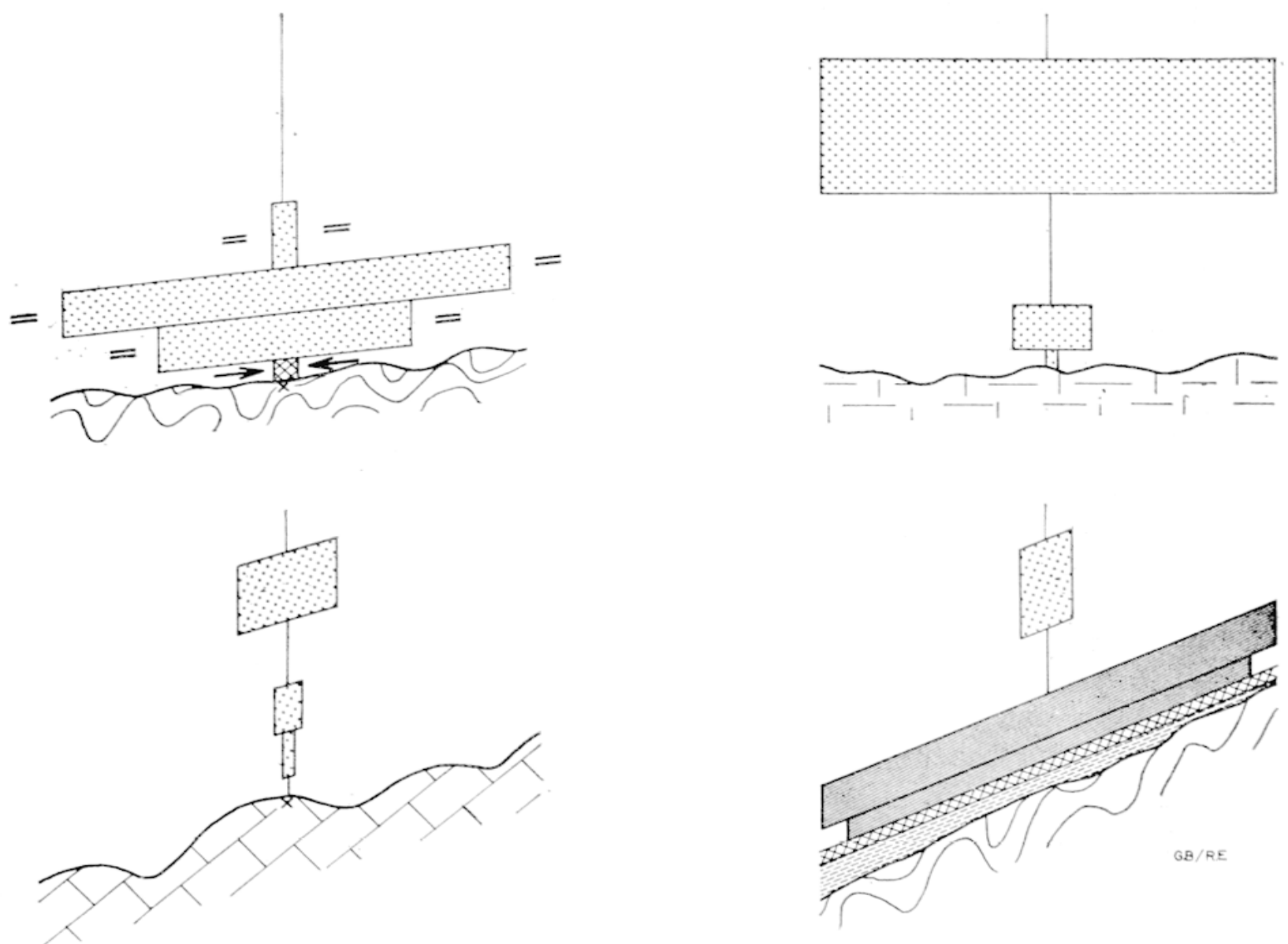

Fig. 10 (en haut, à gauche)

Maquis méditerranéen à Chêne Vert, Filaire et Adénocarpe de la vallée du Quivisa (Liébana, province de Santander, Espagne).

Fig. 11 (en haut, à droite)

Forêt de Pin (reboisement) dans la Serrania de Cuenca
(vallée supérieure du Júcar, Espagne).

Fig. 12 (en bas, à gauche)

Juniperaie à Genévrier Thurifère de la soulane calcaire de Toaca (rio Esla, province du Léon, Espagne).

Fig. 13 (en bas, à droite)

Lande basse subalpine à Ericacées et Genêt (G. trichomanès) du Puerto de Los Senales près Riano ( $1400 \mathrm{~m}$, Monts cantabriques, Espagne). 
Pour étudier l équilibre d'une formation régétale et ses rapports avec l'érosion, il faut done considérer en premier lieu l' "ouverture » ou la « fermeture »du tapis végétal au niveau du sol. Dans la zone tempérée ou la zone tropicale humide, les formations ouvertes sont presque exclusivement des formations secondaires appartenant à des séries régressives. C'est le cas de la garrigue méditerranéenne. Par contre, dans les autres régions du globe. la plupart des formations climaciques sont ourertes, c'est-à-dire que l'érosion est un facteur écolog:que permanent qui, associé à dautres phénomènes, devient un élément fondamental de la répartition et de la dynamique du tapis végétal. C'est, par exemple, le cas de la formation elaire de Genérrier Thurifère qui colonise les hautes soulanes du versant sud cantabrique et dont le développement est perturbé par les éboulis et les cônes d'avalanches actifs (fig. 13).

Cette situation d'équilibre pase le délicat problème des rapports dynamiques entre la végétation et l'érosion «biologique ». L'érasion est un facteur de mobilité écologique. Ises ravinements, les décapages entraînent la disparition du sol et de la litière, la migration des substances colloïdales, l'assechement biologique du substratum. Ise complexe absorbant s'appaurit. Isa végétation ne peut plus se régénérer normalement. Les espèces exigeantes disparaissent. Le tapis végétal se modifie. Isa Subéraie de riébana (fig. 14) est une tormation relicte à l'équilibre précaire (10). I.es plantules de Chêneslièges poussent mal, la forêt abattue ne se reconstitue pas et la isse la place à un maquis d'Arbousier. I a surpécoration et les incendies ourrent le tapis végétal, les sols sont érodés et le terme de l'évolution, certainement irréversible, est une Cistaic à touffes espacées. Grâce à leur enracinement, à leur xérophilie, et surtout à leur incroyable possibilité de multiplication, les Cistes à feuilles de sauge arrivent à s'accrocher sur des pentes instables qui, à la limite. évoluent en badlands. I,érosion devient alors un rigoureux « farteurlimitant ».

Dans les rapports entre une formation végétale et l'érosion «biologique » il faut distinguer deux cas :

- la végétation l'emporte sur l'érosion. Vine formation fermée, climacique ou non (fig. 13), ne donne aucune prise à l'érosion (11). A la suite d'une catastrophe naturelle (incendie, chablis) ou plus fréquemment $d$ 'une intervention humaine, la couverture régétale se déchire, l'érosion peut apparaître et se développer. Maais au bout d'un temps plus on moins long et par dynamique naturelle, la végétation va re-coloniser l'espace vide, d'abord par l'intermédiaire

(10) G. Beatrund, Esquisse biogégraphique de la Liébana (Espagne). Hev. géogr. Pyr. et S.O., 1964, fasc. 3, pp. 225-262. 
d'espèces spécialisées, qui préparent le retour de la formation climax. I. érosion «biologique » conserve alor's le caractère d un érénement exceptionnel. C'est le eas de la plupart des formations végétales de la zone tempérée et de la zone tropicale humide.

- lérosion l'emporte sur la dynamique régétale tt assure la distribution et la mobilité des formations régétales. I cixemple le plus net est colui de la colonisation des éboulis mobiles (12). Mais on trouve de nombreux exemples de cettc subordination do la régétation à l'érosion dans les régions arides et somi-arides (steppe à touffes des glacis plus on moins actifs du sud tunisien).

En se plaçant du point de vue biogéographique, l'éresion apparait comme un facteur écologique essentiel que l'on a trop négligé. Il peut y aroir érosion « biologique » sous forêt, même dans la zone tempérée, à condition que les strates intérieures soicnt ouvertes. Du point de vue géomorphologique il est possible de mieux comprendre comment l'érosion peut se déclencher de faron épidermique. sous couvert végétal et s’étendre ensuite jusqu’à destruction plus ou moins complète de la régétation. Cette sorte de démarrage au ralenti, au sein d'une formation régétale en déséquilibre écolog:que, permettra peut-être, un jour, de mieux comprendre comment s'effectue le passage des phases de biostasie aux phases de rhexistasie.

la méthode des pyramides présente enfin $u$ ecrtain intérêt pour l'étude des ressants. Il est souvent difficile et toujours délicat de repéror sur une pente la nature, l’intensité et la fréquence d'un processus d'érosion (13). De ce fat, l'étude quantitative est souvent illusoire. Par contre, il est toujours possible d obscrver la régétation, du moins ce qu'il en reste. Ia densité du tapis régétal, la localisation des plantes, la sélection naturelle des espeeces et la mobilité vépéale, le moke d enrarinement et le «déchaussement » des collets, l'état de la litière etc. fournissent autant d indications sur la dynamique des rersants.

Cette méthode indirecte étayée sur des relovés floristiques très précis, en particulier au niveau des strates inféricures, et sur la construction de promides régétales détaillées, est en cour's d'application sur le versant sud cantahrique et dans les Aspres du Roussillon. Flle offe lavantage deetudier le romplexe d'erolution du versant dans toute son intégrité.

(11) Voir cependant le cas de resosion sous foret tropicalo ombrophile : G. Rovgrif, Le faconnement actuel des modeles en Cite-divoire forestiere. Mem. I. F. A. N., no $58,1960,542$ p.

(12) J. Bravi-Blasquet, La végetalion alpine el nime des Alpes franģaises. Et. botan. etage alpin, Vii Congrès intcrnat. Botanique, Bayeux, 19it.

(13) J. Tнгс⿰丿丶⿸⿴巳一丶 Paris, Masson, 1962. 
ReVue géographigue des Pyrénéles et di S.-O., $t$. XXXVII, 1966. - PI. II.

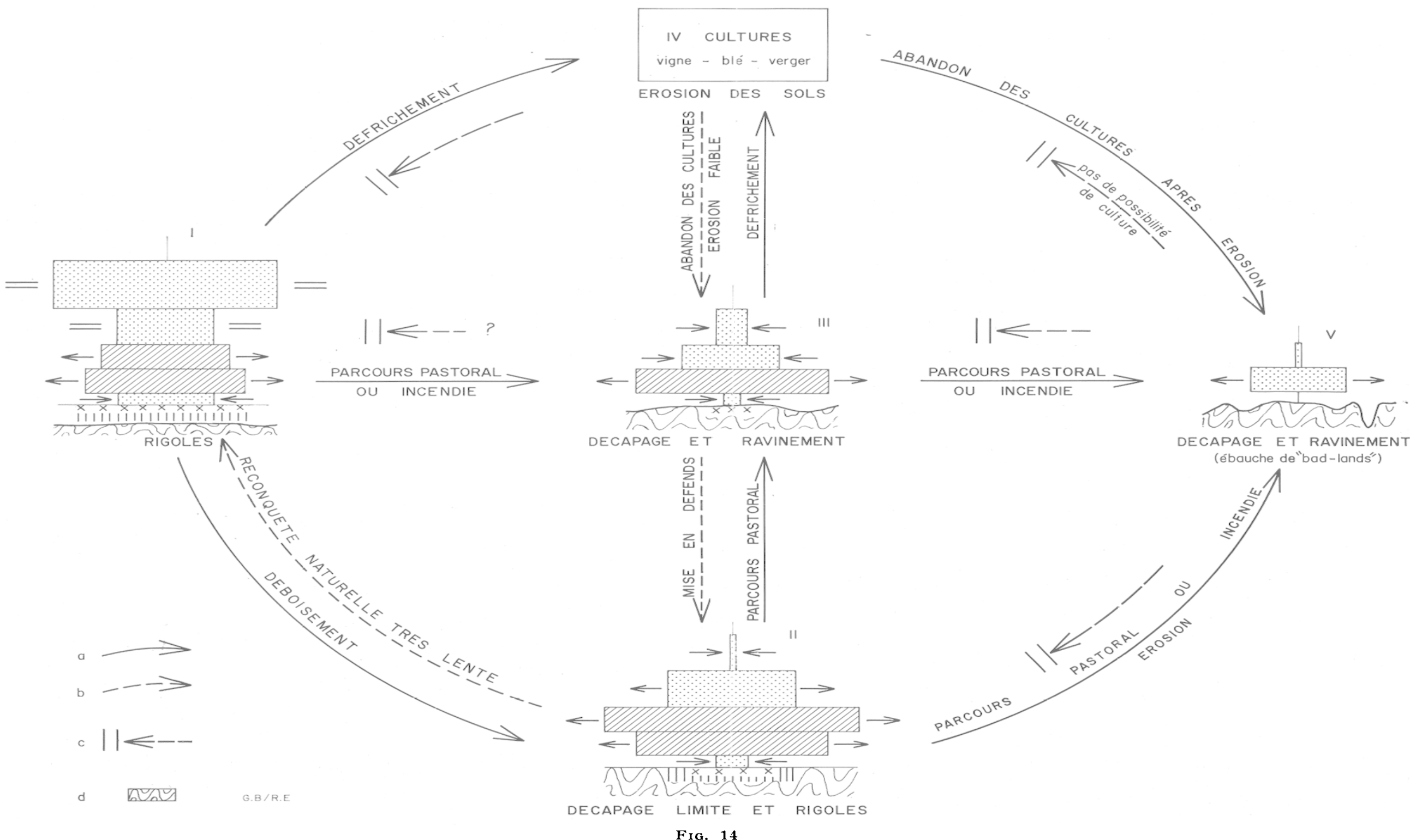

Dynamique de la Subéraie de Liébana (Province de Santander).

2. Etages : 1. Subéraie claire à sous-bois d'Arbousiers sur sol brun et Mistes.- 5. Formation ouverte à Cistes à feuilles de sauge et à Lavande pédonculée.

d. Sehistes en plaquettes. 